\title{
ReaR
}

ISNN 19894090

Revista electrónica de AnestesiaR

Noviembre 2021

CASOS CLÍNICOS

\section{Síndrome de Takotsubo tras traumatismo que requiere intervención quirúrgica urgente.}

Vicente Orgaz M, Gholamian Ovejero S, González-Velasco R, Gómez Del Pulgar Vázquez B.

Hospital Universitario Severo Ochoa, Leganés, Madrid.

\section{Resumen}

El síndrome de Takotsubo (STT), es una miocardiopatía de estrés que afecta más frecuentemente a mujeres postmenopaúsicas tras un estímulo físico o emocional. Se caracteriza por presentar una clínica similar al síndrome coronario agudo, con elevación de enzimas cardiacas y alteraciones en el electrocardiograma. El diagnóstico se basa en los "InterTAK Diagnostic Criteria" establecidos en 2018, siendo fundamental el hallazgo de una anormalidad en la contracción del ventrículo izquierdo, sin patología coronaria (aunque su aparición no excluye el diagnóstico), con reversibilidad del cuadro precoz. El tratamiento no está claramente establecido, incluyendo medidas de soporte y la prevención de complicaciones.

En los últimos años, ha habido un aumento de su descripción en los entornos sanitarios, lo que sugiere un probable infradiagnóstico. El entorno perioperatorio puede ser un escenario ideal para su aparición, por la implicación del estrés quirúrgico, siendo el anestesiólogo el principal implicado en su diagnóstico y manejo.

\section{Introducción}

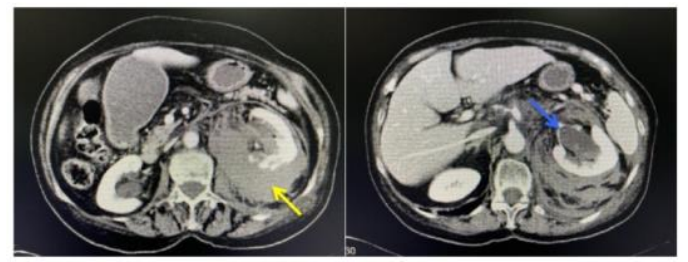

El síndrome de Takotsubo (STT), es una miocardiopatía de estrés que afecta más frecuentemente a mujeres postmenopaúsicas tras un estímulo físico o emocional. Se caracteriza por presentar una clínica similar al síndrome coronario agudo, con elevación de enzimas cardiacas y alteraciones en el electrocardiograma. $\mathrm{El}$ diagnóstico se basa en los "InterTAK Diagnostic Criteria" establecidos en 2018, siendo fundamental el hallazgo de una anormalidad en la contracción del ventrículo izquierdo, sin patología coronaria (aunque su aparición no excluye el diagnóstico), con reversibilidad del cuadro precoz. El tratamiento no está claramente establecido, incluyendo medidas de soporte y la prevención de complicaciones.

En los últimos años, ha habido un aumento de su descripción en los entornos sanitarios, lo que sugiere un probable infradiagnóstico. El entorno perioperatorio puede ser un escenario ideal para su aparición, por la implicación del estrés quirúrgico, siendo el anestesiólogo el principal implicado en su diagnóstico y manejo.

El síndrome de Takotsubo (STT), es una miocardiopatía de estrés descrita por primera vez en 1990 en Japón ${ }^{1-2}$. Se presenta con más frecuencia en mujeres postmenopáusicas tras un estrés físico o emocional, siendo la estimulación simpática exagerada una causa fundamental de este síndrome ${ }^{3 .}$ 
La presentación clínica es indistinguible de un síndrome coronario agudo (SCA), incluyendo la elevación de enzimas cardiacas y cambios en el electrocardiograma $(\mathrm{ECG})^{2-3}$.

El diagnóstico se realiza en base a la clínica acompañada de unos hallazgos típicos descritos en la ecocardiografía, sin evidencia de enfermedad coronaria ${ }^{3}$.

En los últimos años se ha observado un aumento del número de casos, también en el periodo perioperatorio. Será en este contexto donde el anestesiólogo deberá diagnosticar y manejar dicha patología, descartando siempre el SCA por sus implicaciones pronósticas $\mathrm{y}$ terapéuticas ${ }^{4}$.

Se presenta este caso clínico, con la aprobación por parte de la paciente, con el objetivo de ampliar el conocimiento y manejo de esta cardiomiopatía infrecuente, que puede presentarse con el estrés quirúrgico y que se trata de un diagnóstico de exclusión.

\section{Material y métodos}

Mujer de 79 años de edad, sin antecedentes personales de interés, que acude a Urgencias por traumatismo costal izquierdo secundario a caída desde su propia altura, cursando con dolor de difícil control. En la radiografía de tórax se evidencia fractura de la $11^{\circ}$ costilla izquierda y en el ECG, ritmo sinusal a $100 \mathrm{lpm}$.

A las 4 horas de su admisión comienza con mal estado general, hipotensión y aumento del dolor en hemitórax e hipocondrio izquierdo. Al repetir el ECG, se objetiva elevación de $>2 \mathrm{~mm}$ del segmento ST y onda $\mathrm{T}$ picuda en derivaciones precordiales V2-V4 $\mathrm{y}$, elevación $>1 \mathrm{~mm}$ en I, aVL, V1 y V5 (figura 1). Se extrae analítica, destacando: acidosis metabólica, troponina $\mathrm{T} 111 \mathrm{ng} / \mathrm{dL}$ y ácido láctico $4.61 \mathrm{mmol} / \mathrm{L}$.

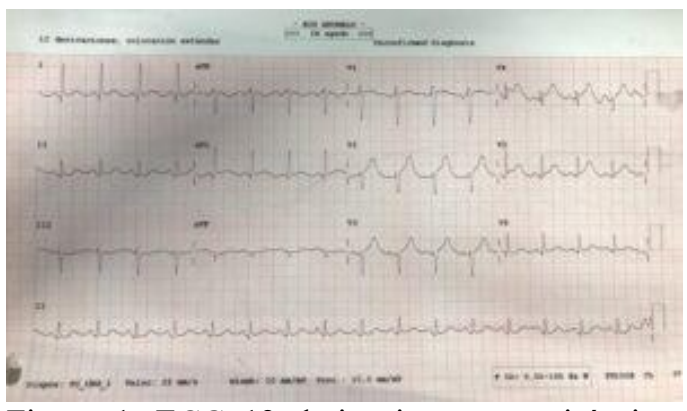

Figura 1. ECG 12 derivaciones prequirúrgico: Ritmo sinusal a $100 \mathrm{lpm}$. Elevación de $>2 \mathrm{~mm}$ del segmento ST y onda $\mathrm{T}$ picuda en derivaciones precordiales V2-V4 y, elevación $>$ $1 \mathrm{~mm}$ en I, aVL, V1 y V5. Fuente: autores.

Se decide realizar una tomografía computarizada abdomino-pélvica urgente objetivando una contusión renal izquierda con defectos de repleción en pelvis renal en relación con hematoma interno, e imagen de extravasación de contraste desde la misma. A nivel pulmonar, aumento de densidad basal izquierda en relación con atelectasia o contusión pulmonar (figura 2).

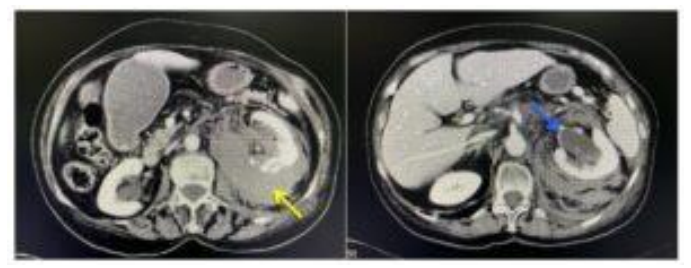

Figura 2 a y b: Cortes transversales de tomografía computarizada abdominal con contraste intravenoso, donde se objetiva: -Hallazgos compatibles con contusión renal izquierda con afectación del sistema colector grado III vs IV de la AAST (American Asociation for the Surgery of Trauma). (Flecha amarilla)

-Pelvis renal con defectos de repleción en probable relación con hematoma interno e imagen de extracción de contraste desde pelvis renal. (Flecha azul) Fuente: autores.

Con estos datos, se activa el protocolo de hemorragia masiva y se interviene de forma urgente. Se realiza una anestesia general con inducción de secuencia rápida, destacando inestabilidad hemodinámica secundaria a la pérdida masiva de sangre ( $\approx 1.8$ litros $)$, que 
requiere politransfusión sanguínea con hemoderivados, reposición de iones e inicio de noradrenalina como vasopresor.

Destacan como hallazgos quirúrgicos, un hematoma renal izquierdo extenso encapsulado junto con laceración esplénica por lo que se realiza una nefrectomía total izquierda y esplenectomía.

Finalizada la intervención, se traslada a la paciente a la Unidad de Reanimación, donde se realiza un ECG de 12 derivaciones para constatar los hallazgos electrocardiográficos, y un ecocardiograma transtorácico, observando: hipertrofia septal asimétrica, obstrucción dinámica del tracto de salida del ventrículo izquierdo (TSVI), insuficiencia mitral (IM) moderada grado III/IV secundaria a movimiento anómalo válvula mitral (SAM), aquinesia de los segmentos medio-distales del septo y distales de cara anterior, inferior y ápex con contractilidad conservada, en base a hipercontractilidad compensadora del resto de segmentos (figura 3 a y b).

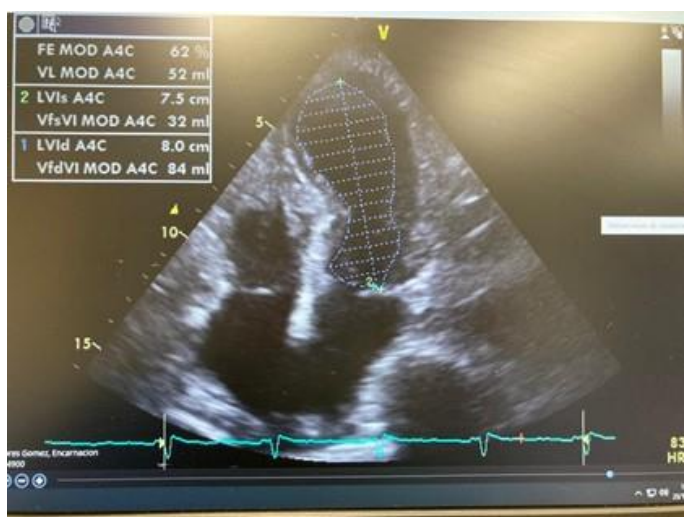

Figura 3a: Ecocardiograma en proyección apical 4 cámaras al final de la sístole el día del ingreso. La FEVI se calculó en $62 \%$. Se muestra la dilatación del ventrículo izquierdo en fase aguda. Fuente: autores.

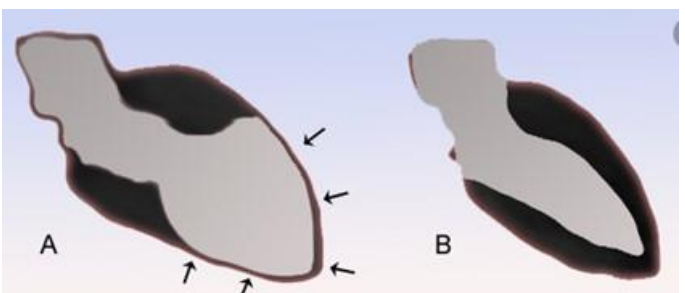

Figura 3b: Representación esquemática de la miocardiopatía de Takotsubo (A) comparada con un paciente normal (B). Fuente: autores.

Dada la alta sospecha de SCA se inicia tratamiento con antiagregantes $\mathrm{y}$ betabloqueo, sin descartar otras posibilidades plausibles: a) un trombo autolisado en alguna o varias ramas de la arteria coronaria izquierda $\mathrm{y}, \mathrm{b}$ ) un STT secundario al traumatismo.

La paciente permanece 6 días en la Unidad de Reanimación, siendo extubada al $2^{\circ}$ día postoperatorio y necesitando soporte respiratorio (ventilación mecánica no invasiva, gafas nasales de alto flujo e incentivo respiratorio) debido a la contusión pulmonar. La inestabilidad hemodinámica fue corregida pudiendo retirar la noradrenalina al $4^{\circ}$ día (dosis máximas de $0.4 \mathrm{mcg} / \mathrm{kg} / \mathrm{min}$ ). A nivel cardiológico, la seriación de troponinas mostraba una discordancia entre su elevación y la imagen observada en la ecocardiografía (110-340-762-765 $\mathrm{ng} / \mathrm{dL})$. Se realizaron dos ecocardiogramas más, uno a los 3 días de ingreso y otro al alta, mostrando imágenes similares y fracción de eyección del ventrículo izquierdo conservada. Esto conllevó finalmente al diagnóstico de STT, descartándose SCA tras una coronariografía normal. Finalmente, se dio de alta a los 7 días iniciando tratamiento con calcioantagonistas (verapamilo).

\section{Discusión}

El STT, también conocido como balonamiento apical del ventrículo izquierdo (VI), síndrome del corazón roto, aturdimiento miocárdico, 
cardiomiopatía inducida por estrés o síndrome de discinesia apical transitoria, fue descrito por primera vez en Japón por Sato et al en $1990^{1}$. El motivo de esta nomenclatura es la semejanza existente entre el VI en sístole y la vasija que utilizan los pescadores japoneses para atrapar pulpos ${ }^{2}$.

Se estima que representa del 1-3\% del total de los SCA, afectando en un $90 \%$ a mujeres de edad media (67-70 años $)^{2}$.

Aunque su fisiopatología no está clara, una de las teorías se centra en el aumento excesivo de catecolaminas durante el estrés emocional o físico, que activa los receptores beta $\mathrm{y}$ alfa adrenérgicos, causando espasmo microvascular que eventualmente precipita la lesión de los miocitos ${ }^{2,3}$.

Otra teoría, dada la notable afectación de mujeres postmenopáusicas, hace sospechar que la disminución de los niveles de estrógenos podría aumentar la susceptibilidad. El estrógeno induce la producción de proteína de choque térmico y péptido natriurético auricular, los cuales se cree que son cardioprotectores contra los efectos adversos del aumento repentino de catecolaminas y el estrés oxidativo ${ }^{2-4}$.

Por lo descrito anteriormente, existe la posibilidad de que varios factores de riesgo predispongan a su aparición, entre ellos: factores hormonales, genéticos, psiquiátricos, neurológicos, emocionales y físicos ${ }^{2-4}$.

La sintomatología más predominante es dolor torácico agudo, disnea o síncope, indistinguible de un SCA a primera vista. También pueden aparecer síntomas derivados de las complicaciones como insuficiencia cardíaca, edema agudo de pulmón (EAP), accidente cerebrovascular, shock cardiogénico o paro cardíaco ${ }^{2-4}$.
Se pueden diferenciar varios tipos de STT en función del lugar de afectación de la discinesia, siendo el más común el globo apical ${ }^{2-4}$.

Su evolución habitual es benigna y reversible, pero no está exenta de morbimortalidad. Hasta un $20-25 \%$ de los pacientes puede desarrollar obstrucción del TSVI y/o IM aguda. Los factores asociados son: edad avanzada, hipertensión arterial, hipertrofia septal, SAM de la válvula mitral e inestabilidad hemodinámica ${ }^{5}$.

Los criterios diagnósticos fueron revisados en 2018 por un comité de expertos internacional, y publicados con el nombre "InterTAK Diagnostic Criteria", realizándose los siguientes cambios con respecto a los criterios de la Clínica Mayo del 2010: 1) se incluye el feocromocitoma como causa desencadenante (previamente criterio de exclusión), 2) se añade la posibilidad de enfermedad arterial coronaria concomitante (10-29\% de los casos), 3) si la presencia de anormalidad en la motilidad parietal del VI compromete el territorio de una arteria coronaria (normalmente descendente anterior), se debe realizar RM cardiaca para descartar SCA y miocarditis ${ }^{6,7}$. Tabla 1 a.

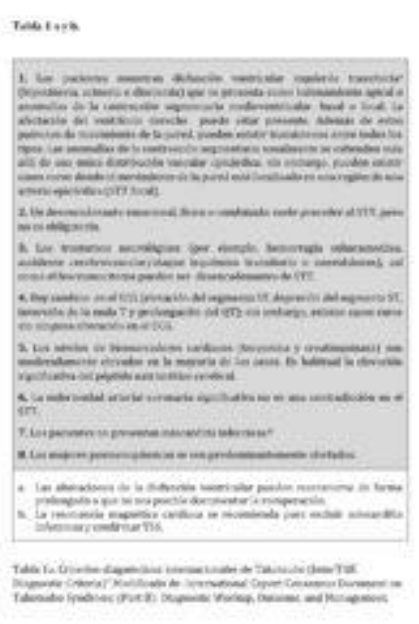


Tabla 1a. Criterios diagnósticos internacionales de Takotsubo (InterTAK Diagnostic Criteria)7.Modificado de: International Expert Consensus Document on Takotsubo Syndrome (Part II): Diagnostic Workup, Outcome, and Management.

Estos criterios comprenden siete parámetros con un máximo alcanzable de 100 puntos. Los pacientes con 30 puntos tienen una probabilidad prevista de $<1 \%$ de sufrir STT, con 50 puntos del $18 \%$ y con $>70$ puntos de $>90 \%{ }^{7}$. Tabla 1 b.

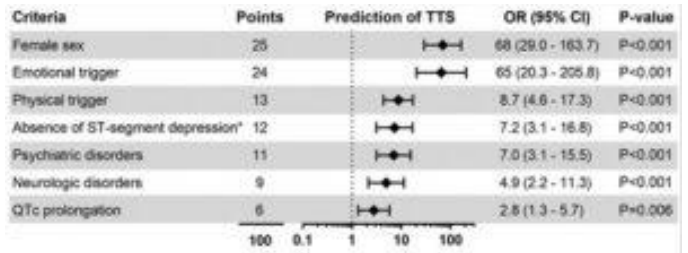

Tabla 1b. Score diagnóstico InterTAK7. Predictores para el diagnóstico de Síndrome de Takotsubo mediante análisis de regresión logística múltiple. Odds ratios de los parámetros de sexo femenino, desencadenante emocional, desencadenante físico, ausencia de depresión del segmento ST, trastornos psiquiátricos, trastornos neurológicos y prolongación del QTc, que se eligieron para construir el InterTAK Diagnostic Score. Modificado de: International Expert Consensus Document on Takotsubo Syndrome (Part II): Diagnostic Workup, Outcome, and Management.

En cuanto al tratamiento del STT, se carece de directrices sobre su manejo y este se basa en la experiencia clínica y el consenso de expertos, centrándose en la fase aguda en un tratamiento de soporte y prevención de complicaciones graves, y tratándose inicialmente como un SCA, hasta descartarlo ${ }^{7,8}$.

Cuando se documenta el STT, los calcioantagonistas tipo diltiazem o verapamilo han resultado ser útiles ${ }^{7,8}$.

En los casos leves, en los que aún no está documentado, podrían usarse anticoagulantes para la prevención de trombos apicales, antiagregantes y estatinas, y en aquellos con fracción de eyección de VI (FEVI) $35-45 \%$, se considerarán los betabloqueantes (BB), y un inhibidor de la enzima convertidora de angiotensina $(\text { IECA })^{9,10}$.

En pacientes más graves, debe realizarse vigilancia estricta en una Unidad de Cuidados Intensivos $\mathrm{O}$ Reanimación durante 72 horas. En pacientes inestables se debe descartar la obstrucción del TSVI, ya que en estos casos está contraindicado el uso de fármacos inotrópicos y el balón de contrapulsación intraaórtico. Sin embargo, el uso de vasoconstrictores (fenilefrina), terapia con volumen y los BB están indicados. Si presenta insuficiencia cardiaca debe ser tratado con IECAS, BB, diuréticos $\mathrm{y}$ vasodilatadores ${ }^{9}$.

En casos con disfunción de VI, parece que el uso de fármacos inotrópicos es dañino por su papel en la génesis de este síndrome ${ }^{7-9}$, aunque algunos informes de casos demuestran mejoría hemodinámica y recuperación de la función ventricular9.

Si no se controla la hipotensión, podría ser necesario un balón de contrapulsación intraaórtico, oxigenación por membrana extracorpórea o dispositivos temporales asistidos por ventrículo ${ }^{9-10}$.

Durante el período perioperatorio, se ha descrito una incidencia de 1/6.700 intervenciones quirúrgicas. Aparece con mayor frecuencia en pacientes con anestesia general $(60 \%)$ y en el periodo postoperatorio (58\% frente al $5 \%$ previo a la inducción $)^{10}$.

En este caso, la presentación del STT fue típica, desarrollándose en una mujer postmenopáusica (criterio 8 InterTAK), tras un evento desencadenante estresante, como es un traumatismo (criterio 2 InterTAK). Aparecieron preoperatoriamente cambios en el ECG como elevación de segmento ST en 
derivaciones precordiales (criterio 4 InterTAK) y la elevación de marcadores enzimáticos fue moderada (criterio 5 InterTAK).

En el ecocardiograma se objetivó discinesia ventricular de distribución típica, no coincidente con ningún área coronaria, y transitoria, con mejoría en las primeras 24 horas (criterio 1 InterTAK). No se presentaron en este caso ni alteraciones neurológicas ni signos de miocarditis infecciosa.

El manejo clínico fue de soporte, añadiendo las medidas de reposición sanguínea, así como drogas vasoactivas para mantener la estabilidad hemodinámica.

En la literatura se describen muy pocos casos de traumatismo como desencadenante del STT. Pensamos que esto puede ser debido a un infradiagnóstico del síndrome en cuestión, por lo que se necesitarían más estudios que pusiesen énfasis en su diagnóstico y posterior tratamiento dirigido $^{11}$.

\section{Conclusiones}

- El síndrome de Takotsubo es una miocardiopatía inducida por estrés que afecta más frecuentemente a mujeres postmenopáusicas.

- Los principales elementos etiológicos son la excesiva estimulación simpática, la disfunción microvascular y los bajos niveles de estrógenos.

- El diagnóstico es de descarte, presentándose con una clínica similar al SCA: imagen típica en la ecocardiografía, coronariografía normal y reversibilidad del cuadro de forma precoz.

- El pronóstico suele ser favorable, desapareciendo las alteraciones anatómicas de forma precoz. Sin embargo, pueden aparecer complicaciones como la obstrucción en el TSVI, IM e insuficiencia cardíaca.

- Faltan ensayos controlados aleatorios de estrategias terapéuticas específicas y, por lo tanto, el manejo del STT se basa en la comprensión de la fisiopatología.

- En el entorno perioperatorio existen múltiples potenciales desencadenantes de estrés, por lo que debemos pensar en este síndrome en un paciente con sintomatología de SCA.

Será necesario realizar investigaciones adicionales para responder a las preguntas con más controversia como: ¿existe predisposición genética?, ¿qué opciones de tratamiento son más específicas?, ¿cuál es la fisiopatología exacta por la que ocurre?, o, ¿por qué hay diferentes fenotipos de STT?

\section{Reconocimientos}

\section{Contribuciones de los autores}

Autor 1 (MVO): Planificación y gestión del caso, búsqueda bibliográfica, interpretación de resultados, redacción inicial y final, aceptación de la responsabilidad por todos los aspectos del trabajo.

Autor 2 (SGO): Planificación del caso, búsqueda bibliográfica, interpretación de los resultados, redacción inicial, aprobación del manuscrito final, aceptación de la responsabilidad por todos los aspectos del trabajo.

Autor 3 (RGV): Búsqueda bibliográfica, redacción inicial, interpretación de los resultados, aprobación del manuscrito final, aceptación de la responsabilidad por todos los aspectos del trabajo. 
Autor 4 (BGPV): Búsqueda bibliográfica, redacción inicial, interpretación de los resultados, aprobación del manuscrito final, aceptación de la responsabilidad por todos los aspectos del trabajo.

\section{Bibliografía}

1. Sato H, Tateishi H, Uchida T, DoteK, Ishihara M, Sasaki K. Takotsubo- like left ventricular dysfunction due to multivessel coronary spasm. Clinical Aspect of Myocardial Injury: from Ischemia to Heart Failure. Tokyo: Kagakuhyoronsha Publishing Co.1990; 56-64. (HTML)

2. Ghadri, J., Wittstein, I., Prasad, A., Sharkey, S., Dote, K., Akashi et al. International Expert Consensus Document on Takotsubo Syndrome (Part I): Clinical Characteristics, Diagnostic Criteria, and Pathophysiology. Eur Heart J. 2018; 39(22):2032-46. doi: 10.1093/eurheartj/ehy076. (PubMed)

3. Wittstein IS, Thiemann DR, Lima JA, Baughman KL, Schulman SP, Gerstenblith $G$ et al. Neurohumoral features of myocardial stunning due to sudden emotional stress. $\mathrm{N}$ Engl J Med. 2005;352:539-48. doi: 10.1056/NEJMoa043046. (PubMed)

4. Rodriguez A, Freire L, Cerizola M, Olivera M, Cordobez J, Trujillo P. Síndrome de takotsubo durante la inducción de una anestesia general. Anes, Analg Reanim. 2012;25(1):1318. (HTML)

5. Schlack E, Aranda F. Miocardiopatía de Takotsubo y movimiento sistólico anterior de válvula mitral. Rev Chil Anest. 2020; 49: 581-85. doi: $10.25237 /$ revchilanestv49n04.17. (PDF)

6. Madhavan M, Prasad A. Proposed Mayo Clinic criteria for the diagnosis of Tako-Tsubo cardiomyopathy and long-term prognosis. Herz. 2010;35(4):240-43. doi: 10.1007/s00059-010-3339-x. (PubMed)

7. Ghadri, J., Wittstein, I., Prasad, A., Sharkey, S., Dote, K., Akashi et al. International Expert Consensus Document on Takotsubo Syndrome (Part II): Diagnostic Workup, Outcome, and Management. Eur Heart J. 2018; 39(22):2047-62. doi: 10.1093/eurheartj/ehy077. (PubMed)

8. Prasad, A., Lerman, A. and Rihal, C.Apical ballooning syndrome (TakoTsubo or stress cardiomyopathy): A mimic of acute myocardial infarction. Am Heart J. 2008;155(3):408-17.doi: 10.1016/j.ahj.2007.11.008. (PubMed)

9. Hessel, E.A. Takotsubo cardiomyopathy and its relevance to anesthesiology: a narrative review. J Can Anesth. 2016;63:1059-74. doi.10.1007/s12630-016-0680-4. (PubMed)

10. Agarwal S, Sanghvi C, Odo N, Castresana MR. Perioperative takotsubo cardiomyopathy: Implications for anesthesiologist. Ann Card Anaesth. 2019;22:309-15. doi: 10.4103/aca.ACA 71_18. (HTML)

11. Síndrome de takotsubo secundario a traumatismo Raquimedular. Rev Colomb Cardiol. 2018;25 (3):237.e1237.e5.

doi.10.1016/j.rccar.2017.07.012. (

Correspondencia al autor

Marta Vicente Orgaz

martavicenteorgaz@hotmail.com

Facultativo especialista adjunto Anestesiología, Reanimación y Terapéutica del Dolor.

Hospital Universitario Severo Ochoa, Leganés, Madrid (España).

Aceptado para el blog en septiembre de 2021 